\title{
Effect of the time-of-day of training on explicit memory
}

\author{
F.F. Barbosa and F.S. Albuquerque \\ Departamento de Fisiologia, Universidade Federal do Rio Grande do Norte, Natal, RN, Brasil \\ Correspondence to: F.F. Barbosa, Departamento de Fisiologia, Universidade Federal do Rio Grande do \\ Norte, Lagoa Nova, Caixa Postal 1511, 59072-970 Natal, RN, Brasil \\ Fax: +55-84-211-9206. E-mail: ffb@natal.digi.com.br
}

\begin{abstract}
Studies have shown a time-of-day of training effect on long-term explicit memory with a greater effect being shown in the afternoon than in the morning. However, these studies did not control the chronotype variable. Therefore, the purpose of this study was to assess if the time-of-day effect on explicit memory would continue if this variable were controlled, in addition to identifying the occurrence of a possible synchronic effect. A total of 68 undergraduates were classified as morning, intermediate, or afternoon types. The subjects listened to a list of 10 words during the training phase and immediately performed a recognition task, a procedure which they repeated twice. One week later, they underwent an unannounced recognition test. The target list and the distractor words were the same in all series. The subjects were allocated to two groups according to acquisition time: a morning group ( $\mathrm{N}=32)$, and an afternoon group $(\mathrm{N}=36)$. One week later, some of the subjects in each of these groups were subjected to a test in the morning $(\mathrm{N}=35)$ or in the afternoon $(\mathrm{N}=33)$. The groups had similar chronotypes. Long-term explicit memory performance was not affected by test time-of-day or by chronotype. However, there was a training time-of-day effect $[F(1,56)=53.667 ; P=0.009]$ with better performance for those who trained in the afternoon. Our data indicated that the advantage of training in the afternoon for long-term memory performance does not depend on chronotype and also that this performance is not affected by the synchronic effect.
\end{abstract}

Key words: Memory; Circadian rhythms; Explicit memory; Recognition memory

Research supported by a fellowship from CNPq (\#132509/2005-9). The authors received financial support from the Department of Physiology and the Psychobiology Program of the Universidade Federal do Rio Grande do Norte.

Received August 22, 2007. Accepted May 6, 2008

\section{Introduction}

There are various studies showing the modulation of circadian rhythmicity in different cognitive processes, including memory (1-3). Among the forms of assessing how biological rhythms may influence memory, the most classic is to study the time-of-day effect on the performance of subjects in different memory tasks (4-6). Regardless of which methodology is applied, it must be remembered that memory is not a unitary system, which makes investigations that attempt to understand how this modulation occurs much more complex. Memory can be divided into two large systems according to subjacent neural substrate and behavioral aspects: declarative or explicit memory and non-declarative or implicit memory (7). The first system depends on the hippocampus for the consolidation pro- cess and memory recall occurs consciously, i.e., recalling episodes of our life. The second system is independent of the hippocampus and is expressed unconsciously, i.e., habits, associative learning, non-associative learning, etc. $(7,8)$.

Folkard et al. $(4,5)$ studied the time-of-day effect on the explicit memory of young individuals using recognition and free recall tests. They reported better performance in the morning tests than in those performed in the afternoon in cases in which recall occurred a short time after training, that is, for short-term memory. When recall occurred a long time after training, there was a training time-of-day effect. In this case, the subjects who acquired information in the afternoon had better performance than those who acquired it in the morning. Interestingly, there was no test time-of-day effect, which suggests circadian modulation 
only in the acquisition or consolidation processes, or in both.

An important aspect in the study of circadian rhythmicity modulation in memory is the chronotype of the participants. Chronotype represents an individual difference in temporal organization of the sleep-wake cycle, i.e., goingto-sleep and wake-up times and the phase of greatest arousal (9-12). There are different instruments to classify individuals according to their chronotype, among them, one developed by Horne and Östberg (9). In this questionnaire, the subjects are classified according to scores relative to questions into five different categories: extreme morning type, moderate morning type, intermediate type, moderate afternoon type, and extreme afternoon type. It is suggested that the chronotype of a subject may change over the course of life and studies indicate that in childhood (between 8 and 12 years) there is a tendency to being a morning type, whereas in adolescence and in the early adult phase, there is a tendency to being an afternoon type, returning to the morning type tendency in old age $(12,13)$.

Studies that used memory tasks with a short retention interval have shown a so-called synchronic effect, which consists of better performance when subjects of a determinant chronotype perform the task at their optimal time-ofday (13-16). An important implication of these chronotype studies is that perhaps the training time-of-day effect on long-term explicit memory reported by Folkard et al. (4) may have been, in fact, a consequence of the sample that was used. The subjects in these studies were adolescents or young undergraduates, a population with a greater tendency to being afternoon types. Thus, the better afternoon performance may have been a synchronic effect, that is, a better acquisition of information in the afternoon owing to the larger number of afternoon types in the sample. Testu and Clarisse (6) repeated this research with children, adding to the investigation a possible influence of day of the week on which the test occurred (Monday or Thursday). However, they arrived at different results. Shortterm memory performed better when the test took place in the afternoon, only on Thursday, and they found no acquisition time-of-day effect on long-term memory. They did, however, find a recall time-of-day effect, which was better in the morning. Interestingly, the sample used in their study was younger, composed of children between 8 and 10 years of age, which is a population likely consisting of more morning types. Perhaps the different results obtained in the two studies are due to the difference in age of the two samples.

Considering the importance of chronotype in the study of circadian rhythmicity modulation, the main purpose of the present research was to determine if the reported timeof-day of training effect on explicit memory is due to the chronotype of the subjects, that is, evaluate a possible synchronic effect of chronotype on long-term explicit memory.

\section{Material and Methods}

A total of 68 undergraduate students (28 men and 40 women) aged 18 to 25 years $(21 \pm 2)$ took part in the study after giving written informed consent to participate without financial remuneration. The project was approved by the Institutional Ethics Committee. The subjects were allocated to three groups according to their score on the Horne-Östberg ( $\mathrm{HO})$ Portuguese version questionnaire (17). The moderate and extreme morning types were classified as morning types and the moderate and extreme evening types were classified as evening types. Twentythree subjects (9 men and 14 women) were classified as morning types (HO score: $66 \pm 6$ ), 23 (9 men and 14 women) were classified as intermediate types (HO score: $51 \pm 4$ ), and 25 (10 men and 12 women) were classified as evening types (HO score: $37 \pm 4$ ).

\section{Task procedure}

The task was applied individually. In the acquisition phase (training), each subject listened to a list of ten words through a set of headphones. Immediately afterwards, they were given the recognition test, in which the subject listened to 20 words, 10 from the target list and 10 distractor words in random order. The subjects responded "yes" if the word was on the list they had just heard; otherwise, they responded "no" if the word was a distractor. The subject had $5 \mathrm{~s}$ to respond. Both target and distractor words were concrete Portuguese language nouns and both sets contained a similar number of syllables and semantic categories. After the first recognition test, the procedure (acquisition test) was repeated twice, in sequence, in order to permit the subjects to learn the target list. One week later, the students returned to the laboratory for an unexpected long-term memory (LTM) test. They were presented with twenty words, and again they answered a recognition test. The target and distractor words were the same in all series. The use of the same distractor words in the series was done in order to create proactive interference in the recognition test after a one-week interval. The responses of all tests were recorded by the same evaluator.

\section{Experimental design}

The subjects were allocated to two groups according to the training phase: one group in the morning $(\mathrm{M})$, between 
7:30 and 9:30 $\mathrm{h}(\mathrm{N}=32)$ and the other in the afternoon $(\mathrm{A})$, between 16:30 and 18:30 h $(\mathrm{N}=36)$. One week later some of the students in each group took the LTM test in the morning ( $N=35$, groups $M M$ and $A M)$ or in the afternoon $(N$ $=33$, groups $\mathrm{MA}$ and $\mathrm{AA})$. Each group consisted of 3 chronotypes and a similar number of subjects from all chronotypes (Table 1).

\section{Statistical analysis}

The subject's performance in the LTM test was used as a dependent variable. The measure of performance was obtained by subtracting the total number of false alarms from the total number of hits, a common measure of recognition memory (18). "Yes" responses to items belonging to the list were considered hits while false alarm was considered for "yes" answers to distractor words. We used ANOVA with 3 factors: 3 (chronotype) x 2 (training time) $\times 2$ (LTM test time). The $t$-test for independent samples was the post hoc test used and the significance level was $\mathrm{P} \leq 0.05$ for all analyses.

\section{Results}

The LTM performance variation of the subjects did not show a main effect of test time-of-day or of chronotype [F $(1,56)=0.249, P=0.620 ; F(2,56)=0.238, P=0.789$, respectively]. However, we found a main effect of training time-of-day $[\mathrm{F}(1,56)=7.376, \mathrm{P}=0.009]$. Post hoc analysis indicated better performance in the afternoon than in the morning $(P=0.009)$. The interactions between the factors were not statistically significant [chronotype $x$ test time-of- day: $F(2,56)=0.425 ; P=0.656$; chronotype $x$ training time-of-day: $F(2,56)=0.837, P=0.438$; test time-of-day $x$ training time-of-day: $F(1,56)=1.014, P=0.318$; chronotype $x$ test time-of-day $x$ training time-of-day: $F(2,56)=1.509, P$ $=0.230 ;$ Table 1].

\section{Discussion}

The main findings in this study were: 1) training time-ofday effect on long-term explicit memory, the afternoon being better than the morning, regardless of chronotype; 2) no interaction between LTM test time-of-day and chronotype, indicating the absence of a synchronic effect.

The main purpose of this study was to determine if the training time-of-day effect on explicit memory previously reported by Folkard et al. $(4,5)$ could be explained, at least in part, by the chronotype of the participants. The subjects of these studies were mostly young, a population with a greater tendency to being afternoon types $(13,14)$. In this case, it could be suggested that the better performance of individuals who trained in the afternoon was a result of a synchronic effect and not related to training time-of-day (13-15). However, even when the chronotype variable was controlled, the result was the same. The subjects who acquired information in the afternoon performed better, even though one-third of the sample consisted of subjects classified as morning types. A plausible explanation for this result is that most studies that show an interaction between chronotype and cognitive task time-of-day applied memory tests with a short interval between training and the test (13-15). Perhaps, previously consolidated

Table 1. Experimental design and performance of subjects in this study.

\begin{tabular}{|c|c|c|c|c|c|c|c|}
\hline \multirow[t]{3}{*}{ Test time } & \multirow[t]{3}{*}{ Chronotype } & \multicolumn{4}{|c|}{ Training time } & \multicolumn{2}{|r|}{ Total } \\
\hline & & \multicolumn{2}{|r|}{ Morning } & \multicolumn{2}{|r|}{ Afternoon } & \multirow[b]{2}{*}{$\mathrm{N}$} & \multirow[b]{2}{*}{ Mean \pm SEM } \\
\hline & & $\mathrm{N}$ & Mean \pm SEM & $\mathrm{N}$ & Mean \pm SEM & & \\
\hline \multirow[t]{4}{*}{ Morning } & Morning & 6 & $5.167 \pm 1.352$ & 6 & $3.833 \pm 0.703$ & & \\
\hline & Evening & 5 & $2.800 \pm 1.158$ & 6 & $4.500 \pm 1.285$ & & \\
\hline & Intermediate & 6 & $3.000 \pm 1.095$ & 6 & $6.000 \pm 1.033$ & & \\
\hline & Total & & & & & 35 & $4.257 \pm 0.466$ \\
\hline \multirow[t]{4}{*}{ Afternoon } & Morning & 5 & $1.800 \pm 0.969$ & 6 & $5.000 \pm 0.856$ & & \\
\hline & Evening & 5 & $3.400 \pm 1.249$ & 6 & $4.667 \pm 1.406$ & & \\
\hline & Intermediate & 5 & $2.800 \pm 0.800$ & 6 & $5.667 \pm 1.282$ & & \\
\hline & Total & & & & & 33 & $4.000 \pm 0.489$ \\
\hline Total & & 32 & $3.219 \pm 0.466^{*}$ & 36 & $4.944 \pm 0.442^{*}$ & & \\
\hline
\end{tabular}

Data are reported as mean \pm SEM of performance (hits minus errors). Three-way ANOVA was carried out. Post hoc $t$-test indicated subjects performed better in the afternoon than in the morning. ${ }^{*}$-value $=$ -2.69 (d.f. $=66$ ); $P=0.009$. 
long-term memory recall is not affected by the synchrony between time-of-day and best performance time of the subject. To the best of our knowledge, the present study was the first to evaluate a possible relationship between chronotype and performance on a long-term explicit memory test. In addition to corroborating previous studies, our data refined these findings by consistently indicating that the training time-of-day effect seems to be independent of chronotype and of the task used $(4,5)$.

In accordance with Folkard et al. (5), the present study also found no interaction between training time-of-day and test time-of-day. Studies of rodents have found that memory recall at the same time of day in which it was acquired favors the recall process. This phenomenon has been shown more than once, both in positive and negative modified reinforcement protocols $(3,19,20)$ and was denominated "temporal stamp" by Ralph et al. (3). This may occur because of a mechanism similar to that of statedependent learning (5). However, it seems that the acquisition time-of-day effect for LTM performance was greater than that originating from the same state during the test, or it could be that this effect is very subtle and could not be detected in the present protocol.

The purpose of using the same distractor words in all series was to create proactive interference in the long-term recognition test. Since all words in this test had already been codified in LTM, they were somewhat familiar (21). This protocol enabled us to determine if the capacity to resist proactive interference (22) could vary according to both training time-of-day and test time-of-day. The results reinforce the advantage of acquiring information in the afternoon, considering that the performance measure used was the number of hits minus the number of errors.

There are studies showing a positive correlation between measures of operational memory span and the capacity of subjects to resist interference $(22,23)$. One of the theoretical implications of these studies is that good operational memory function may lead to less interference. This could occur through distinct mechanisms. One of these might be through improved information integration during learning, leading to better codification and, in turn, less interference during long-term memory recall. A second mechanism would be that a less efficient operational memory could have difficulty in controlling a large flow of information during recall, creating interference (24). Ramirez et al. (2) studied the components of operational memory in subjects submitted to a constant routine protocol and showed that performance improved over the course of the active phase. It can, therefore, be suggested that the higher number of errors is due to inefficient or non-optimized functioning of operational memory. One possibility is that codification in the morning is more susceptible to proactive interference, generating a mnemonic trace without a "signature" that indicates whether the item belongs to the list or is a distractor word. Thus, better performance in the afternoon than in the morning may be due to a circadian variation in the codification mechanisms of explicit memory. Another mechanism, non-exclusive to the previous one, is better consolidation of explicit memory when it is acquired in the afternoon. Ellenbogen et al. (25) showed that sleep improves the performance of subjects in a cued recall test compared to the control group. However, this improvement was much better when an associative interference protocol was used. The authors propose that sleep protects the declarative (or explicit) memory of the interference process. Thus, it is plausible to suggest that the subjects who acquired information in the afternoon had a shorter interval between the test and going to sleep, and derived greater advantages from the role of sleep in memory consolidation. However, it could be expected that the morning type subjects would be benefited more than the afternoon types in the present study, since the interval between information acquisition in the afternoon and going to sleep is shorter in the morning types than in the afternoon types. However, we found no interaction between chronotype and training time-of-day in long-term memory recognition.

It can be concluded that there seems to be no synchronic effect on long-term explicit memory, at least with the protocol used. Therefore, the training time-of-day effect on longterm explicit memory does not depend on the chronotype of the subjects, given that this effect persisted even when this variable was controlled. Further studies are needed to determine if this effect is a result of a circadian variation in the acquisition process or if it is due to differences in the consolidation and storing mechanisms of explicit memory.

\section{Acknowledgments}

We would like to thank all volunteers, without whose participation this study could have not been carried out, and Dr. Regina H. da Silva for her invaluable comments. 


\section{References}

1. Maheu FS, Collicutt P, Kornik R, Moszkowski R, Lupien SJ. The perfect time to be stressed: a differential modulation of human memory by stress applied in the morning or in the afternoon. Prog Neuropsychopharmacol Biol Psychiatry 2005; 29: 1281-1288.

2. Ramirez C, Talamantes J, Garcia A, Morales M, Valdez P, Menna-Barreto L. Circadian rhythms in phonological and visuospatial storage components of working memory. Biol Rhythm Res 2006; 37: 433-441.

3. Ralph MR, Ko CH, Antoniadis EA, Seco P, Irani F, Presta C, et al. The significance of circadian phase for performance on a reward-based learning task in hamsters. Behav Brain Res 2002; 136: 179-184.

4. Folkard S, Monk TH, Bradbury R, Rosenthall J. Time of day effects in school children's immediate and delayed recall of meaningful material. Br J Psychol 1977; 68: 45-50.

5. Folkard S, Monk TH. Circadian rhythms in human memory. Br J Psychol 1980; 71: 295-307.

6. Testu F, Clarisse R. Time-of-day and day-of-week effects on mnemonic performance. Chronobiol Int 1999; 16: 491503.

7. Squire LR, Zola SM. Structure and function of declarative and nondeclarative memory systems. Proc Natl Acad Sci U $S$ A 1996; 93: 13515-13522.

8. Squire LR, Kandel ER. Memory: from mind to molecules. New York: W.H. Freeman and Company; 2000.

9. Horne JA, Ostberg O. A self-assessment questionnaire to determine morningness-eveningness in human circadian rhythms. Int J Chronobiol 1976; 4: 97-110.

10. Roenneberg T, Wirz-Justice A, Merrow M. Life between clocks: daily temporal patterns of human chronotypes. $J$ Biol Rhythms 2003; 18: 80-90.

11. Mongrain V, Lavoie S, Selmaoui B, Paquet J, Dumont M. Phase relationships between sleep-wake cycle and underlying circadian rhythms in morningness-eveningness. J Biol Rhythms 2004; 19: 248-257.

12. Kim S, Dueker GL, Hasher L, Goldstein D. Children's time of day preference: age, gender and ethnic differences. Pers Individ Dif 2002; 33: 1083-1090.

13. May CP, Hasher L. Synchrony effects in inhibitory control over thought and action. J Exp Psychol Hum Percept Per- form 1998; 24: 363-379.

14. Intons-Peterson MJ, Rocchi P, West T, McLellan K, Hackney A. Age, testing at preferred or nonpreferred times (testing optimality), and false memory. $J$ Exp Psychol Learn Mem Cogn 1999; 25: 23-40.

15. Intons-Peterson MJ, Rocchi $\mathrm{P}$, West $\mathrm{T}$, McLellan K, Hackney A. Aging, optimal testing times, and negative priming. $J$ Exp Psychol Learn Mem Cogn 1998; 24: 362-376.

16. Natale V, Alzani A, Cicogna P. Cognitive efficiency and circadian typologies: a diurnal study. Pers Indiv Dif 2003; 35: 1089-1105.

17. Benedito-Silva AA, Menna-Barreto L, Marques N, Tenreiro $\mathrm{S}$. A self-assessment questionnaire for the determination of morningness-eveningness types in Brazil. Prog Clin Biol Res 1990; 341B: 89-98.

18. Lockhart RS. Methods of memory research. In: Tulving $E$ (Editor), The Oxford handbook of memory. Oxford: Oxford University Press; 2000. p 45-72.

19. Cain SW, Chou T, Ralph MR. Circadian modulation of performance on an aversion-based place learning task in hamsters. Behav Brain Res 2004; 150: 201-205.

20. Ko $\mathrm{CH}$, McDonald RJ, Ralph MR. The suprachiasmatic nucleus is not required for temporal gating of performance on a reward-based learning and memory task. Biol Rhythm Res 2003; 34: 177-192.

21. Wilson SP. The development of efficient inhibition: evidence from directed-forgetting tasks. Dev Rev 1998; 18: 86-123.

22. Friedman NP, Miyake A. The relations among inhibition and interference control functions: a latent-variable analysis. $J$ Exp Psychol Gen 2004; 133: 101-135.

23. Bunting M. Proactive interference and item similarity in working memory. J Exp Psychol Learn Mem Cogn 2006; 32: 183-196.

24. Radvansky GA, Copeland DE. Memory retrieval and interference: working memory issues. J Mem Lang 2006; 55: 3346.

25. Ellenbogen JM, Hulbert JC, Stickgold R, Dinges DF, Thompson-Schill SL. Interfering with theories of sleep and memory: sleep, declarative memory, and associative interference. Curr Biol 2006; 16: 1290-1294. 